\title{
Actuales condiciones pedagógicas para el desarrollo del módulo de Informática Contable en los institutos no gubernamentales de El Progreso, Yoro
}

\section{Current pedagogical conditions for the development of the Accounting Informatics module in the non-governmental institutes of El Progreso, Yoro}

Jeffry Saúl Rivas ${ }^{1}$

\section{Resumen}

El propósito de esta investigación es describir las condiciones pedagógicas actuales en que se desarrolla el módulo de Informática Contable, especialmente, las condiciones de infraestructura, el perfil docente que atiende el módulo, condiciones didácticas y la valoración que hace el estudiante sobre el desarrollo del módulo desde la perspectiva de su aprendizaje. La investigación se hizo con un enfoque cuantitativo y se analizaron nueve variables que permitieron llegar a conclusiones más precisas y obtener información que sirve de base fundamental para la elaboración de una propuesta, la cual consiste en estrategias de gestión sobre elementos de infraestructura, estrategias didácticas y de evaluación; por último, se proponen estrategias de selección y profesionalización docente. Estas estrategias tienen como propósito mejorar la calidad de los procesos de enseñanza aprendizaje en el módulo de Informática Contable.

Palabras clave: Informática contable, condiciones de infraestructura, perfil docente, condiciones didácticas, estrategias educativas

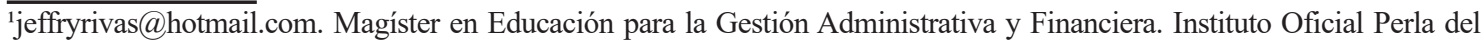
Ulúa. Honduras. https://orcid.org/0000-0003-0330-6251
} 


\begin{abstract}
The purpose of this research is to describe the current pedagogical conditions in which the Accounting Informatics module is developed, especially, the infrastructure conditions, the teaching profile that attends the module, didactic conditions and the assessment that the student makes about the development of the module from the perspective of his learning. The research was carried out with a quantitative approach and nine variables were analyzed that allowed to reach more precise conclusions and obtain information that serves as the fundamental basis for the elaboration of a proposal, which consists of management strategies on infrastructure elements, teaching and evaluation strategies; finally, strategies for selection and professionalization of teachers are proposed. These strategies are intended to improve the quality of the teaching-learning processes in the Accounting Informatics module.
\end{abstract}

Keywords: Accounting informatics, infrastructure conditions, teacher profile, didactic conditions, educational strategies

\title{
Introducción
}

La contabilidad e informática son recursos básicos que proporcionan información importante en cualquier toma de decisión, la utilización de estos recursos de manera eficiente aporta una mejora continua en todos los procesos administrativos de una organización empresarial. Por otra parte, el software contable es un recurso que ha hecho eficiente los procesos de registro, almacenamiento, procesamiento y presentación de la información contable y financiera en el momento que se requiere.

Debido a la importancia de las Tecnologías de la Información y Comunicación es que dentro de los procesos de formación en carreras afines a la administración general, la contabilidad y las finanzas; se han incorporado en los planes de estudio módulos curriculares de informática donde los estudiantes adquieren las competencias básicas de informática, búsqueda y selección de información; de igual manera competencias el uso de hojas de cálculos como el Excel u otros programas de Microsoft Office. 
Con la implementación de los nuevos Bachilleratos Técnicos Profesionales en el sistema educativo hondureño en el año 2013 (PRAEMHO, 2007), se hicieron diversos cambios que originaron nuevos módulos curriculares dentro de la formación de los estudiantes. En estos módulos curriculares se asignaron o se reubicaron docentes sin tener las competencias en el área de la informática, área administrativa, contable y financiera; que son las que le permiten al docente planificar, diseñar, guiar y orientar procesos de enseñanza aprendizaje, y de evaluación dentro del módulo de Informática Contable. Esto condiciona la capacidad de la planificación pedagógica, metodológica y didáctica de los procesos de enseñanza aprendizaje, ya que resulta difícil según Perdomo (2016) que un docente tenga un dominio "riguroso de lo que enseña y de la ciencia didáctica que posibilita llevar a cabo la pedagogización del conocimiento y las experiencias de aprendizaje" (p.105).

También es importante destacar la falta de recursos didácticos que tienen la mayoría de institutos no gubernamentales para desarrollar procesos de enseñanza aprendizaje en el módulo de Informática Contable. Para demarcar esta situación se ha podido observar que algunos institutos no cuentan con suficientes recursos informáticos ni libros de texto que se puedan utilizar para desarrollar los contenidos curriculares; por lo tanto, se dificulta la ejecución de actividades de enseñanza y aprendizaje que incluya prácticas contables usando como recurso un software contable; además, no se puede llevar a cabo un proceso de evaluación de los aprendizajes haciendo uso de los recursos informáticos.

Es preciso señalar que según estudio exploratorio con los docentes, se ha identificado que los estudiantes "no tienen una base sólida de conocimientos previos en contabilidad, finanzas, matemáticas e informática" (J. Hernández, comunicación personal, 08 de febrero del 2018). Esto constituye una dificultad para los procesos de adquisición del aprendizaje en el módulo de Informática Contable; ya que de alguna manera se obliga a realizar una readecuación pedagógica y didáctica corriendo el riesgo de que el tiempo no sea lo suficiente para cubrir todos los contenidos que constituyen la competencia del módulo.

La investigación parte de una evaluación curricular y es pertinente en el marco del Currículo Nacional Básico y está en las líneas de estudio de la Universidad Pedagógica 
Nacional Francisco Morazán (UPNFM) dentro del sistema educativo nacional en los cambios o reformas curriculares hechas por el sistema educativo formal. Además, los resultados de esta investigación podrían servir de marco referencial, proporcionando una base de conocimiento para realizar futuras investigaciones en diferentes contextos. Los resultados que generó esta investigación, podrían servir de base para que las autoridades de la Secretaría de Educación y de los centros educativos tomen decisiones en beneficio de la calidad educativa. Se identificaron necesidades de capacitación de los docentes y la investigación sirvió de base para elaborar una propuesta con el fin de mejorar la calidad de la educación y garantizar la adquisición de las competencias profesionales por parte de los estudiantes.

\section{Objetivos de Estudio}

\section{Objetivo General}

Analizar las actuales condiciones pedagógicas para el desarrollo del módulo de Informática Contable en el duodécimo grado de Bachillerato Técnico Profesional en Contaduría y Finanzas, de los institutos no gubernamentales de El Progreso, Yoro, durante el I semestre del año 2018.

\section{Objetivos Específicos}

1. Identificar las condiciones de infraestructura en las que se desarrolla el módulo de Informática Contable.

2. Describir el perfil del docente que atiende el módulo de Informática Contable en los institutos no gubernamentales de El Progreso, Yoro.

3. Describir las condiciones didácticas en las que se desarrolla el módulo de Informática Contable.

4. Identificar la valoración que hacen los estudiantes sobre el desarrollo del módulo de Informática Contable, considerando las condiciones de infraestructura, perfil docente y condiciones didácticas.

5. Proponer una serie de estrategias para mejorar la calidad del proceso de enseñanza aprendizaje en el módulo de Informática Contable. 


\section{Discusión Teórica}

Los autores como Coll (1992), Grundy (1998), Stenhouse (1991), Zabalza (2000), Taba (1976), Arnaz (1981) aporta importantes lineamientos teóricos sobre la teoría curricular que sirven de sustento para el diseño del currículo escolar en cualquier sistema educativo. El currículo como recurso normativo orienta las prácticas educativas porque contiene “una declaración de finalidades o de objetivos específicos, una selección y organización de contenidos, ciertas normas de enseñanza y aprendizaje y un programa de evaluación de resultados" (Taba, 1976, citado por Diaz, Gónzales, Pacheco, Saad, \& Rojas, 2008, p.18).

El docente es el responsable de poner en práctica el currículo y de realizar ciertas readecuaciones debido a los cambios o necesidades del entorno educativo. Ante esta situación, el currículo tiene que permanecer "abierto a una discusión crítica que pueda ser trasladado efectivamente a la práctica educativa" (Meza, 2012, p. 8); asimismo, el currículo tiene que ser evaluado para diagnosticar sus fortalezas, debilidades y facilitar toma de decisiones. Esta evaluación curricular se debe realizar para saber si se cumple lo diseñado y "determinar la conveniencia de conservarlo, modificarlo o sustituirlo" (Díaz, 1990, p. 133).

La evaluación del currículo se considera una tarea de investigación en el marco de las ciencias sociales, con una perspectiva vinculada a la evaluación de una parte del currículo, donde se reconoce al evaluador como un investigador, que está obligado a construir el objeto de estudio y elaborar la fundamentación conceptual con que realizará la indagación del objeto de estudio y entregará los resultados que será un informe de investigación (Díaz, 2005).

El presente estudio parte de una evaluación curricular propuesta por Díaz Barriga, 2005, como modelo de investigación, donde la autora establece tópicos puntuales que le permite al investigador enfocarse en un objeto de estudio específico dentro de los fundamentos de un currículo y del funcionamiento de un plan de estudios. Para llevar a cabo la evaluación se sustenta con lineamientos de los Planes y Programas de Estudio de Educación Media 
especialmente del programa del Bachillerato Técnico Profesional en Contaduría y Finanzas (PRAEMHO, 2007), además, del Manual de Puestos y Salario Docentes de la Secretaría de Educación donde se describe el perfil docente y las competencias requeridas para desempeñar funciones de docencia (Secretaría de Educación, 2017).

Otra de las líneas teóricas que respaldan la investigación, es las Tecnologías de la Información y Comunicación (TIC) como recurso didáctico para el aprendizaje de los contenidos del módulo curricular; de igual manera, la teoría sobre los elementos del proceso didáctico citados por Medina y Mata, 2009; Mattos, 1974; y la evaluación de los aprendizajes por Pimienta, 2008; Pimienta 2012; Díaz y Hernández, 2007; que sirven de fundamento teórico en el proceso de la investigación y en la elaboración de las estrategias propuestas en el presente estudio.

\section{Condiciones de infraestructura}

Una de las estructuras fisicas que debe tener todo centro educativo para el desarrollo del proceso de enseñanza aprendizaje es el Laboratorio de Computación, que según Nakazawa et al., (2018) es "el espacio educativo construido dentro de un inmueble dotado de equipamiento y los medios necesarios para la enseñanza y el aprendizaje de la informática" (p.43). Son recursos tecnológicos que en la mayoría de los casos se les asocia potenciales pedagógicos, didácticos y tecnológicos; además, se utilizan en la enseñanza y aprendizaje del uso y manejo de las Tecnologías de la Información y Comunicación (TIC).

El mobiliario del Laboratorio de Computación debe estar diseñado de acuerdo a las características físicas del estudiante, ya que "el tamaño físico del cuerpo, interviene en el diseño del mobiliario; pues debe posibilitar donde apoyar la espalda, piernas, cabeza, brazos, y que permita el rozamiento con otras superficies, ya que estos son elementos que actúan como estabilizadores del cuerpo" (Panero \& Zelnik, 1998, citado en Flores et al., 2017, p.32).

Otro elemento importante de infraestructura es el acceso a Internet, ya que por muchas razones es el medio de información más democrático que existe en la actualidad. Permite 
enviar y recibir mensajes por correo electrónico; colocar un objeto de información en un espacio virtual con una dirección específica, de modo que el resto de los individuos conectados a Internet puedan acceder a él (Semenov, 2005, p.60). Es necesario tener acceso a Internet en los Laboratorios de Cómputo o centro educativo, ya que el docente debe hacerlo útil como recurso de aprendizaje. Según Valzacchi (2003) el Internet como recurso de aprendizaje es útil porque permite que el docente colabore con otros a través de foros de discusión en donde puedan intercambiar sus experiencias y mejorar el desempeño, también se puede encontrar material y recurso didáctico con el que se pueda trabajar en el aula; y por último se puede encontrar información complementaria de una actividad de enseñanza aprendizaje y nuevas oportunidades de formación profesional para el docente.

\section{El Software del Módulo de Informática Contable}

En el plan curricular del módulo de Informática Contable se sugieren recursos didácticos como las hojas de cálculo en Microsoft Excel, software de contabilidad (Mónica 8.5) y la página electrónica de declaración de impuestos DET-Live (PRAEMHO, 2007). Un software contable "permite registrar y procesar las transacciones generadas de una actividad económica, sean ventas, compras, créditos; logrando sistematizar y simplificar dichas operaciones aplicando los cálculos necesarios para sus registros efectivos que orientan el manejo adecuado de las transacciones de una empresa” (Pico \& Núñez, 2018, p. 23).

\section{Perfil del Docente}

La formación inicial del docente debe de estar acorde a los cambios apresurados de la sociedad y que le permita en la práctica un mejor desempeño de sus funciones, ya que una formación inicial fuera del contexto histórico y social por si misma resulta "insuficiente al ser contrastada con los cambios acelerados que se dan en la sociedad ya que presentan exigencias que requieren de la investigación, el trabajo colaborativo y la innovación como vías integradas al desempeño docente" (Perdomo, 2016, p.96). La formación docente tiene que tener un enfoque curricular basado en competencias, pues una formación docente basada en competencias es aquella que integra los tres saberes; "el saber conocer, el saber hacer y el saber ser" (Tobón, 2005, p. 174). Con este enfoque el docente tiene los conocimientos, 
habilidades y destrezas para llevar a cabo la práctica educativa, asimismo, demuestra en su quehacer educativo tener los valores, principios, conductas y actitudes que de alguna manera influye en la formación integral de los educandos. La formación inicial y permanente del docente debe de estar enmarcada en el profesionalismo autónomo, innovador, flexible, participativo, con alto grado de liderazgo, donde pueda escoger con fundamentos los métodos que considera más idóneo para sus alumnos (Avalos \& Nordenflycht, 1999).

\section{Acompañamiento Pedagógico}

Es un espacio formativo para el docente que puede recibir de un grupo de personas especialistas en el área educativa durante sus prácticas pedagógicas; de esta manera el acompañamiento pedagógico es un proceso que "orienta, dialoga, cuestiona, confronta con resultados, ayuda a ver debilidades y fortalezas; recuerda compromisos acordados, propone alternativas, anima y asegura la continuidad de los planes" (Hurtado \& Paredes, 1999, citado en Carrasco, 2009, p.33). Además, el acompañamiento pedagógico es un proceso donde el docente mejora su práctica educativa debido a que se crean "espacios de diálogo, análisis de situaciones, intercambio de ideas, creencias y valores, la crítica constructiva; y las posibilidades teóricos prácticos de proponer, experimentar, interpretar y evaluar las acciones educativas" (Perdomo, 2013, p.74)

\section{Elementos del Proceso Didáctico}

Los elementos del proceso didáctico son el alumno, el maestro, lo objetivos de aprendizaje, los contenidos, los recursos didácticos, los métodos, las técnicas y los procedimientos didácticos (Medina \& Mata, 2009).

El alumno como elemento del proceso didáctico es el factor personal decisivo en la situación escolar; es activo y emprendedor; para él se organiza la escuela y se administra la enseñanza; los profesores están a su servicio, para orientarlo e incentivarlo en su educación y en su aprendizaje, con el fin de desenvolver su inteligencia y formar su carácter y personalidad (Mattos, 1974). Para Raymond Wheeler (citado en Mattos, 1974, p. 7) el profesor "es un técnico en ingeniería humana, ya que él es el principal responsable de la modelación de la 
inteligencia y de la personalidad de sus alumnos." Actualmente, el docente "actúa como elemento que estimula, orienta y controla el aprendizaje de los alumnos, adaptando la enseñanza a su capacidad real y a sus limitaciones, aclarando sus dudas y ayudándoles en sus vacilaciones y dificultades" (Mattos, 1974, p. 31).

Los contenidos son "el conjunto de saberes o formas culturales cuya asimilación y apropiación por los estudiantes se considera esencial para su desarrollo y socialización" (Coll, 1992, p. 188). En efecto, los contenidos son los conocimientos de las diferentes ciencias, las habilidades, las actitudes y las conductas, es decir, todo aquello que sea objeto de aprendizaje. Los recursos didácticos son los medios de enseñanza que constituyen distintas imágenes y representaciones que se confeccionan especialmente para la docencia; también, abarcan objetos naturales e industriales, tanto en su forma natural como preparada, los cuales contienen información y se utilizan como fuentes de conocimiento, algunos de ellos son objetos impresos y medios sonoros o de proyección informáticos; por ejemplo, las hojas de trabajo, colores, juego geométrico, papel milimétrico, película, presentación en Power Point, libro de texto, discos compactos, modelos, etcétera (Pimienta, 2007).

Por otra parte, el método didáctico "orientan, muestran el camino, el sendero a seguir, nos permiten trazar un rumbo en busca de un objetivo, una meta, una finalidad o un fin" (Torres \& Girón, 2009, p. 57). Ahora bien, las técnicas "son maneras racionales y que la experiencia demuestra que son eficaces de conducir una o más fases del aprendizaje escolar y que puede ser llevado a cabo por medio de una serie de procedimientos didácticos" (Mattos, 1974, p. 87). Siguiendo el orden de ideas del mismo autor el procedimiento didáctico "son los segmentos o series de actividades docentes en determinada fase de la enseñanza. Ejemplos: procedimiento de interrogatorio, de demostración, de explicación, de corrección de tareas, organización y aplicación de pruebas objetivas".

\section{Evaluación de los Aprendizajes}

Según Marchesi y Martín (1998, citado en Díaz \& Hernández, 2002) la evaluación permite hacer adaptaciones de las actividades de enseñanza y aprendizaje para proporcionar ayudas 
en el momento que se detectan los problemas, plantear actividades de refuerzo según el grado de consecución de los objetivos y reorientar la planificación de las secuencias de aprendizaje. En la evaluación de los aprendizajes se evalúan conocimientos conceptuales, procedimentales y actitudinales. Los conocimientos conceptuales son los conceptos, hechos y principios de una unidad curricular; los contenidos procedimentales es un conjunto de acciones coordinadas que incluyen métodos, técnicas, procedimientos y habilidades o destrezas para ejecutar una tarea; y finalmente los conocimientos actitudinales son los valores, actitudes y normas compartidas por un grupo de individuos y que se pueden observar en las conductas y comportamientos de las personas (Zabala, 2000). Para evaluar y medir estos conocimientos se debe diseñar y aplicar las técnicas e instrumentos de evaluación que permita identificar y validar los resultados obtenidos con los esperados.

\section{Fases de la Evaluación}

La evaluación inicial tiene un "fin diagnóstico que llevará a tomar decisiones sobre la orientación del proceso al inicio del ciclo escolar” (Pimienta, 2008, p. 38). Identificando los conocimientos o las capacidades cognitivas necesarias para el nuevo programa de aprendizaje y de ser necesario hacer regulaciones pedagógicas para que los conocimientos "puedan ser recuperados intencionalmente en el proceso de enseñanza para establecer relaciones significativas con la información nueva a aprenderse" (Miras, 1993, citado en Díaz \& Hernández, 2007, p. 398).

Por otra parte, evaluación formativa tiene como finalidad "regular el proceso enseñanza y aprendizaje para adaptar o ajustar las condiciones pedagógicas (estrategias y actividades) al servicio del aprendizaje de los alumnos" (Allal et al., 1979, citado en Díaz \& Hernández, 2007, p. 406). Esta fase de de evaluación se realiza supervisando constantemente el desempeño del estudiante en las diferentes actividades de enseñanza y aprendizaje con el fin de identificar aquellas dificultades o errores que conlleve a realizar las modificaciones o adecuaciones en el momento oportuno para beneficio del aprendizaje del alumno. Este tipo de evaluación está dirigida a la mejora continua de los procesos de aprendizaje de los estudiantes y permite 
la retroalimentación de manera constante durante el proceso del aprendizaje; ya que se puede tomar decisiones de restructuración de contenidos, readecuación de la metodología didáctica, intervención para mejorar el clima de aula y visitar o hablar con los familiares del alumno. Es decir, durante el proceso se detecta disfunciones y se ponen remedios que contribuyen a que los procesos de construcción del conocimiento de los estudiantes mejore y no esperar al final donde se puede obtener resultados de reprobación (Pimienta, 2008).

Por último, la evaluación sumativa tiene como fin "verificar el grado en que las intenciones educativas han sido alcanzadas." Provee información con la que se pueden tomar decisiones acerca de una “calificación, acreditación o certificación” (Díaz \& Hernández, 2007, p. 413). Con una serie de técnicas e instrumentos de evaluación se puede recoger datos que permita emitir juicios de valor sobre la validez del proceso y de la situación en que se encuentra cada uno de los alumnos en relación con la consecución de los aprendizajes propuestos. Además, sirve de base para hacer readecuaciones, regulaciones e intervenciones pedagógicas de manera proactivas con los alumnos (Medina \& Mata, 2009).

\section{Técnicas e Instrumentos de Evaluación}

Las técnicas de evaluación son “cualquier instrumento, situación, recurso o procedimiento para obtener información adecuada a los objetivos y finalidades que se persiguen. Además, cada técnica se le debe exigir ciertas características que garanticen al máximo su validez y fiabilidad para evaluar el aprendizaje" (Fernández, 2010, p. 26). Entre las técnicas de evaluación se puede citar la observación de las actividades realizadas por los alumnos, preguntas exploratorias, trabajos y ejercicios hechos en clases y fuera de las clases, los portafolios, mapas conceptuales, evaluación del desempeño, y las pruebas o exámenes escritos (Díaz \& Hernández, 2007).

Los instrumentos de evaluación "son el medio específico que permite la emisión de una valoración" (Pimienta, 2012, p.40), es decir, son los que permiten medir niveles de desempeño ya sea de tipo cualitativo o cuantitativo comparándolos con los productos del aprendizaje, por lo tanto, es importante que el profesor utilice aquellos que permita tener 
una mayor objetividad al momento de medir el desempeño de los estudiantes en algunas actividades o estrategias de aprendizaje. Los instrumentos de evaluación más utilizados son "las rúbricas, los cuestionarios, las guías para evaluar portafolios, las pruebas objetivas, lista de cotejo, guías de observación y los diarios de clases (Pimienta, 2008, p. 29).

\section{Número de Estudiantes por Sección}

El número de estudiantes por sección es un factor muy importante, ya que de ellos depende la interacción en el aula, las oportunidades de aprendizaje y la atención personalizada que hace el maestro con los estudiantes. En los diferentes niveles educativos de Honduras, el número de estudiantes por sección está establecido en el art. No. 26 del reglamento de centros educativos de la Ley Fundamental de Educación, que establece literalmente que:

Las secciones de cada año de estudio del nivel no podrán exceder de treinta (30) educandos de ambos sexos. Cada asignatura de sección deberá ser atendida por un docente que reúna el perfil y los requisitos establecidos en el Manual de Clasificación de Puestos y Salarios. (p.177)

Un estudio hecho por Dockendorff (2015) concluye que actualmente hay consenso en torno a la idea de que los cursos reducidos ofrecen mejores oportunidades de aprendizaje, pero no causan una mejor enseñanza, ya que la relación entre el tamaño del curso y los logros de aprendizaje no es directa y está mediada por procesos de aula intervinientes. Los cursos reducidos permiten una relacion de maximizacion del tiempo de instrucción y la individualización de la enseñanza; al disponer el docente de más tiempo por estudiante producto de una reducción del tiempo dedicado a labores administrativas y de control de la disciplina, las interacciones profesor-alumno aumentan y con ello la frecuencia y calidad de la retroalimentación, facilitando la reflexión y la corrección del error. Además, los cursos reducidos favorecen la visibilidad de todos los estudiantes, afianzan el sentido de pertenencia al grupo y un mayor compromiso hacia el aprendizaje. Los docentes son menos rígidos y punitivos; mejoran la efectividad y calidad de los aprendizajes con conductas dentro del aula que aumentan las estrategias pedagógicas impactando en la satisfacción de los alumnos y docente. 


\section{Los Horarios de Clases.}

Según el art. No.158 de la Ley Fundamental de Educación "la hora clase es el tiempo efectivo que destinan los docentes en función docente, y que la hora clase tiene una duración de 45 minutos" (Secretaría de Educación, 2014, p. 58). Los horarios de clases se hacen en virtud de la cantidad de horas a la semana que tiene cada módulo curricular, la cantidad de horas clases para los Bachilleratos Técnicos profesionales está determinado en los planes y programas de Bachilleratos Técnicos Profesionales diseñado por PRAHEMO.

Marín y Maya, (2015) proponen un modelo de programación de horarios donde se maximicen "la cantidad de bloques por materias, es decir asignaciones de dos horas seguidas de la misma clase, lo que favorece el proceso de aprendizaje, beneficia la agenda de los profesores al disminuir tiempos ociosos entre clases, y favorece los costos y acuerdos contractuales con la institución" (p.47). Este modelo permite un tiempo estimable para desarrollar metodologías didácticas, principalmente aquellas clases que requieren mucho más tiempo para poder explicar ejercicios demostrativos, dar instrucciones, desarrollar actividades de aprendizaje, evaluar y retroalimentar los aprendizajes. La asignación de horarios en bloque de dos horas clases permite la disminución de tiempos muertos entre clases para un mismo docente, el aumento de la eficiencia de las clases ya que no se mezclan muchas materias con poca relación conceptual en un mismo día; y por último, mejora la calidad de estudio de los alumnos por no recibir muchas clases con temas pocos relacionados (Marín \& Maya, 2015).

\section{Métodos y Materiales}

\section{Enfoque de Investigación}

La investigación se realizó desde el enfoque cuantitativo (Hernández, Fernández \& Baptista, 2014); donde se aplicaron técnicas cuantitativas para la recolección de datos y se utilizaron gráficos, tablas y porcentajes para medir, cuantificar, verificar, analizar e interpretar los datos de las nueves variables que fueron objeto de estudio; lo que permitió dar respuesta a las preguntas de investigación y se logró identificar las condiciones de infraestructura, 
describir el perfil docente, describir las condiciones didácticas e identificar la valoración que hacen los estudiantes sobre el desarrollo del módulo de Informática Contable, considerando las condiciones de infraestructura, perfil docente y las condiciones didácticas.

\section{Tipo y Diseño de Investigación}

El tipo de investigación con que se llevó a cabo este trabajo es descriptivo, el cual según Hernández, Fernández, y Baptista, 2014, p. 92 “busca especificar las propiedades importantes de personas, grupos, comunidades o cualquier otro fenómeno que sea sometido a un análisis." Es decir, este tipo de investigación busca caracterizar el objeto de investigación, identificando las variables para una descripción y análisis de los datos. El tipo de diseño de la investigación es no experimental o expost-facto, ya que se realizó sin la manipulación deliberada de variables y en los que sólo se observaron los fenómenos en su ambiente natural para analizarlos, esto es de acuerdo a lo que plantea (Hernández, Fernández, \& Baptista, 2014, p.142).

\section{Variables}

Las variables que fueron objeto de estudio y de análisis en relación a las condiciones de infraestructura son: disponibilidad de mobiliario, equipo de informática y acceso a Internet, condiciones físicas del Laboratorio de Computación, y los programas utilizados en el proceso de enseñanza aprendizaje. En cuanto al perfil docente se estudiaron y analizaron las variables de perfil docente y acompañamiento pedagógico; y en las condiciones didácticas se estudiaron y analizaron los elementos del proceso didáctico, conocimientos previos, número de estudiantes por sección y los horarios.

\section{Población y Muestra}

La población está constituida por 280 estudiantes del duodécimo grado del Bachillerato Técnico Profesional de Contaduría y Finanzas de cuatro institutos no Gubernamentales que son los únicos que ofrecen la carrera en el Municipio de El Progreso, Yoro. También, forman parte de la población cinco docentes que imparten el módulo curricular. En el 
caso de los estudiantes, el diseño de la muestra es probalístico, donde los elementos se seleccionaron de manera aleatoria y se decidió tomar una muestra del 50\% de los estudiantes para tener mayor representatividad de la misma y obtener datos más confiables y precisos. En el caso de los docentes la muestra es no probabilística, y en donde se seleccionaron los cinco docentes, es decir, el 100\% de la población.

\section{Instrumentos de Investigación}

Se utilizaron 3 instrumentos de investigación los cuales pasaron por un proceso de validación, entre ellos un cuestionario que fue aplicado a los estudiantes; una lista de cotejo que se contestó realizando visitas a los laboratorios de computación y al departamento de consejería de cada instituto; además, se realizaron observaciones de las clases para poder contestar algunas preguntas de la lista de cotejo. Por último, se aplicó una entrevista a los docentes con el propósito de obtener datos cualitativos o cuantitativos que permita profundizar y enriquecer los análisis, responder objetivamente las preguntas de investigación, llegar a conclusiones más precisas y que los hallazgos sirvan de fundamento para la propuesta de estrategias orientada a la mejora de la calidad en los procesos de enseñanza aprendizaje.

En el cuestionario se trató en la medida de lo posible solo incluir preguntas cerradas, que "son aquellas que contienen opciones de respuesta previamente delimitadas y resultan más fáciles de codificar y analizar" (Hernández, Fernández, \& Baptista, 2014, p. 127). Con las respuestas del cuestionario se busca conocer la percepción que tienen los estudiantes sobre las variables de disponibilidad de mobiliario y equipo informático, acceso a Internet, condiciones físicas del laboratorio de computación, software utilizado en los procesos de enseñanza aprendizaje, perfil del docente, elementos del proceso didáctico y conocimientos previos con que inician el módulo curricular. Los ítems se agruparon en tres categorías mediante un proceso de recodificación para conocer la dirección de la escala. En este sentido las puntuaciones 1 y 2 se codificaron como "no aceptable", la puntuación 3 como “indeciso" y las puntuaciones 4 y 5 como "aceptable". De acuerdo a los datos de la muestra se presentan seis gráficas con su respectivo análisis; además, por el nivel de significancia 
de algunos datos para esta investigación, se cruzan tablas que ayudan a profundizar el análisis e interpretación de la información obtenida

\section{Análisis de Datos}

Tomando en cuenta, las estrategias de recolección de datos y siguiendo una coherencia metodológica, lo más recomendable para procesar y analizar la información cuantitativa es utilizar una técnica estadística. En este caso, se decidió utilizar SPSS estadístico que “es un paquete estadístico de análisis de datos de aplicación en la investigación de las ciencias sociales y económicas. Contiene programas capaces de realizar desde un simple análisis descriptivo hasta diferentes tipos de análisis multivariante de datos" (Pérez, 2004, p. 16). La información obtenida en las entrevistas se procesó y se analizó por variables, en donde se utilizaron técnicas de reducción, sistematización y conclusiones de los datos.

\section{Resultados}

En este apartado se presentan gráficos de los datos obtenidos del cuestionario, además, se presenta algunas tablas con datos cruzados del cuestionario y de la lista de cotejo con el propósito de profundizar los análisis sobre las variables que son objeto de estudio. También se hace uso de datos cualitativos obtenidos de la entrevista que permite enriquecer los análisis, responder a las preguntas de investigación y llegar a conclusiones más precisas.

\section{Figura 1}

Disponibilidad de mobiliario, equipo de informática y acceso a Internet

\section{$57.9 \%$}

\section{$25.7 \%$}

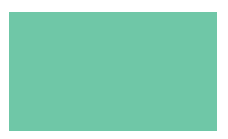

No aceptable
$16.4 \%$

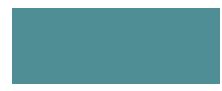

Indeciso

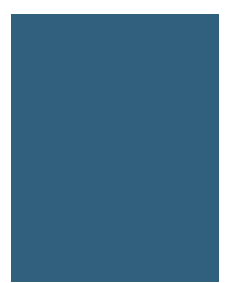

Aceptable 
Tabla 1

Disponibilidad de computadoras y acceso a Internet

\begin{tabular}{lcccc}
\hline \multicolumn{1}{c}{ Instituto } & Sección & $\begin{array}{c}\text { Cantidad de } \\
\text { Estudiantes }\end{array}$ & $\begin{array}{c}\text { Cantidad de } \\
\text { Computadoras }\end{array}$ & $\begin{array}{c}\text { Acceso a } \\
\text { Internet }\end{array}$ \\
\hline José Simón Azcona Hoyo & $12-1$ BTPCF & 28 & 50 & Si \\
Roberto Michelleti Bain & $12-1$ BTPCF & 28 & 28 & No \\
Perla del Ulúa & $12-1$ BTPCF & 47 & 40 & No \\
Perla del Ulúa & $12-2$ BTPCF & 47 & 40 & No \\
Perla del Ulúa & $12-3$ BTPCF & 37 & 40 & No \\
Eduardo Hernández Chevez & $12-1$ BTPCF & 28 & 19 & No \\
Eduardo Hernández Chevez & $12-2$ BTPCF & 27 & 19 & No \\
Eduardo Hernández Chevez & $12-3$ BTPCF & 37 & 19 & No \\
\hline
\end{tabular}

Nota: elaboración propia

El estudio reflejó que más de la mitad (58\%) de los estudiantes están de acuerdo que la disponibilidad de computadoras, el buen estado del mobiliario y el acceso a Internet les ha facilitado los aprendizajes en el módulo de Informática Contable. Pero al relacionar los datos presentados en la Tabla 1 entre los diferentes institutos, encontramos que algunos de ellos no disponen de suficientes computadoras, impresoras, mobiliario del equipo de informática, y acceso a Internet. Esto conlleva a que los estudiantes tengan que compartir computadora y mobiliario generando atrasos en el desarrollo de las actividades de enseñanza aprendizaje; y si a esto le agregamos que un 56\% de los estudiantes no cuentan con computadora personal en su casa, por lo tanto, los estudiantes no tienen otra alternativa que esperar que su compañero termine las actividades para hacer uso 
de la computadora y realizar su propia tarea. Por consiguiente, esto lleva a ocupar más tiempo en el semestre, debido a que no es suficiente para desarrollar la programación de los contenidos que constituyen la competencia profesional del módulo de Informática Contable

En cambio, no tener acceso a Internet en los laboratorios de computación limita la facilidad de buscar información, acceder a videos tutoriales, descargar programas y utilizar plataformas de mensajería o almacenamiento de la información para evitar problemas sobre pérdidas de archivos sin la necesidad de usar dispositivos de almacenamiento externo. Esto debido a que ante la falta de recursos de dispositivos de almacenamiento externo (Memorias USB y Discos de Almacenamiento) ocasiona que los estudiantes pierdan sus trabajos porque los compañeros se los borran y porque las computadoras están programadas con almacenamiento temporal.

No obstante, el estudio reflejó que hay un instituto que cuenta con las condiciones óptimas para el desarrollo del módulo de Informática Contable, ya que tiene 50 computadoras, suficiente mobiliario y acceso a Internet en los Laboratorios de computación.

\section{Condiciones Físicas del Laboratorio de Computación}

Figura 2

Condiciones físicas del Laboratorio de Computación

$79 \%$

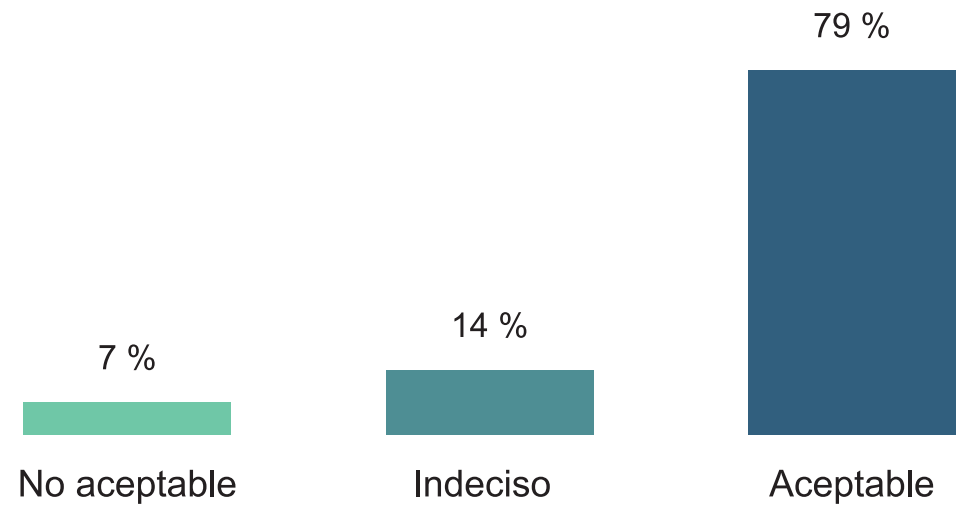


Al consultarles a los estudiantes sobre la condición actual de iluminación, climatización, aspecto de las paredes, techo y pizarra del Laboratorio de Computación, la mayoría (79\%) de ellos está de acuerdo que les ha ayudado a mantener un buen ambiente de aprendizaje en el módulo de Informática Contable. Esto según Herrera (2006 citado en Castro \& Morales, 2015, p. 4) permite tener a disposición del docente y del estudiante "un ambiente de aprendizaje con un entorno físico y psicológico de interactividad regulada en donde confluyen personas con propósitos educativos." Lo que constituye una fortaleza institucional que condiciona el desarrollo integral del estudiante y estimula las buenas relaciones interpersonales entre los actores de la educación.

De igual manera, a consultarle a los docentes afirmaron que los laboratorios de computación tienen buena climatización, instalaciones eléctricas para una adecuada iluminación y conexión de los diferentes dispositivos, se escucha poco ruido y la pizarra está en buenas condiciones. Por lo tanto, las condiciones físicas de los institutos están en óptimas condiciones para crear ambientes o espacios que faciliten el aprendizaje.

\section{Utilización del Software}

Figura 3

Software utilizado en los procesos de enseñanza aprendizaje del módulo de Informática Contable

$80 \%$

$9 \%$

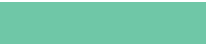

No aceptable
$11 \%$

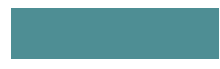

Indeciso

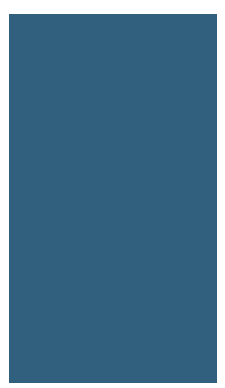

Aceptable 
Tabla 2

Software utilizado en los procesos de enseñanza aprendizaje del módulo de Informática Contable

\begin{tabular}{lccccc}
\hline \multicolumn{1}{c}{ Instituto } & Sección & $\begin{array}{c}\text { Microsoft } \\
\text { Excel }\end{array}$ & $\begin{array}{c}\text { Mónica } \\
8.5\end{array}$ & $\begin{array}{c}\text { Det- } \\
\text { Live }\end{array}$ & $\begin{array}{c}\text { Otro } \\
\text { software }\end{array}$ \\
\hline José Simón Azcona Hoyo & $12-1 \mathrm{BTPCF}$ & $\mathrm{Si}$ & $\mathrm{No}$ & $\mathrm{No}$ & No \\
Roberto Michelleti Bain & $12-1 \mathrm{BTPCF}$ & $\mathrm{Si}$ & $\mathrm{Si}$ & $\mathrm{No}$ & No \\
Perla del Ulúa & $12-1 \mathrm{BTPCF}$ & $\mathrm{Si}$ & $\mathrm{Si}$ & $\mathrm{No}$ & No \\
Perla del Ulúa & $12-2 \mathrm{BTPCF}$ & $\mathrm{Si}$ & $\mathrm{Si}$ & $\mathrm{No}$ & No \\
Perla del Ulúa & $12-3 \mathrm{BTPCF}$ & $\mathrm{Si}$ & $\mathrm{Si}$ & No & No \\
Eduardo Hernández Chevez & $12-1 \mathrm{BTPCF}$ & $\mathrm{Si}$ & $\mathrm{No}$ & No & No \\
Eduardo Hernández Chevez & $12-2 \mathrm{BTPCF}$ & $\mathrm{Si}$ & $\mathrm{No}$ & $\mathrm{No}$ & No \\
Eduardo Hernández Chevez & $12-3 \mathrm{BTPCF}$ & $\mathrm{Si}$ & $\mathrm{No}$ & $\mathrm{No}$ & No \\
\hline
\end{tabular}

Nota: elaboración propia

Ochenta por ciento (80\%) de los estudiantes están de acuerdo que se utilizan programas en el desarrollo de las clases, donde aprenden a hacer transacciones contables, presupuestos y declaración de impuestos. Sin embargo, al relacionar los datos obtenidos en la lista de cotejo y la entrevista personal; en primer lugar se pudo observar que todos los institutos utilizan el programa Microsoft Excel para que los estudiantes realizan actividades de aprendizaje como tablas de amortización, flujos de caja, tablas de depreciación, pronósticos de ventas, planillas; aplican fórmulas financieras (valor futuro, valor presente, valor de la cuota); y solamente en un instituto los estudiantes hacen registros contables y presentan los Estados Financieros utilizando este software.

Al observar los datos de la Tabla 2 solamente dos institutos utilizan el software contable Mónica 8.5 en los procesos de enseñanza aprendizaje. En este software los estudiantes realizan actividades como la creación de una empresa con sus respectivos datos, elaboración de un catálogo de cuentas, creación de inventario de productos, creación de una cartera 
de clientes y proveedores, hacer ordenes de compras, facturas de ventas, generar reportes de ventas, hacer registros en el libro diario, liquidar ciclos contables y presentar los Estados Financieros.

Finalmente, ningún instituto utiliza en los procesos de enseñanza aprendizaje otro software contable que no sea el sugerido por el programa de estudio ni la página electrónica de declaración de tributos Det- Live. De manera general esta situación no es favorable, ya que no se cuenta con recursos o medios educativos actualizados para la enseñanza y aprendizaje de los contenidos que integran la competencia profesional del módulo de Informática Contable, en lo que respecta a contenidos procedimentales tales como "registro de asientos contables, manejo de paquete contable para el registro de transacciones y manejo de hojas electrónicas de declaración de tributos" (PRAEMHO, 2007, p. 543).

\section{Perfil del Docente}

La investigación refleja que un $87 \%$ de los estudiantes están de acuerdo que el docente que imparte el módulo de Informática Contable tiene habilidades o competencias en el uso de la computadora y el software contable; además, demuestra tener conocimientos contables que le ha facilitado el uso del software. También, el docente mantiene buena relación con los estudiantes y se muestra interesado y motivado en el desarrollo de las clases.

Sin embargo, al triangular los datos en relación a la formación inicial del docente, encontramos que solamente tres de ellos son Profesor de educación media en Educación Comercial en el Grado de Licenciatura y el resto tiene una formación de Profesor de Educación Media en Informática Educativa en el Grado de Licenciatura. Es decir, todos tienen formación pedagógica y didáctica para ejercer la docencia, pero con orientaciones diferentes del conocimiento. Ahora bien, al revisar el programa curricular y el Manual de Clasificación de Puestos y Salarios Docente de la Secretaría de Educación el perfil recomendado para impartir el módulo de Informática Contable es el Profesor de Educación Comercial por su amplia formación administrativa, contable, financiera y manejo de las 
tecnologías de la información y comunicación; que le permite planificar, orientar, dirigir, ejecutar y evaluar actividades de aprendizaje donde se utiliza la tecnología para el registro de transacciones generadas por operaciones de tipo contable y financiera que realizan las empresas. Por lo tanto, el Profesor de Educación Comercial tiene "dominio del plan de estudios y dominio teórico y metodológico del área de su competencia" (Secretaría de Educación, 2017, p. 37).

Otro aspecto importante, es que ningún docente está en algún espacio de profesionalización, es decir, nadie está estudiando o participando en programas de formación docente. Por otra parte, el docente mantiene buena relación con los estudiantes basados en el respeto, la confianza, la libertad, la buena comunicación y el compromiso, asimismo, se sienten motivados impartiendo la clase, porque lo consideran un reto personal y profesional, debido a que es una oportunidad de seguir aprendiendo vinculando la parte contable con la informática; igualmente, son conscientes que los estudiantes al egresar debe tener los conocimientos o competencias que le permita ingresar con éxito al mercado laboral.

Figura 4

Perfil del docente que atiende el módulo de Informática Contable

$87 \%$

$9 \%$

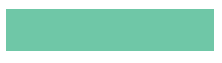

No aceptable
$4 \%$

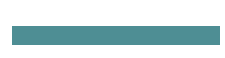

Indeciso

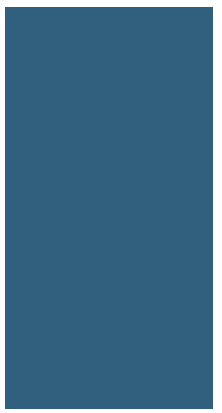

Aceptable

\section{Acompañamiento Pedagógico}

El acompañamiento pedagógico está lejos de cumplir sus objetivos en beneficio del desarrollo profesional del docente y del aprendizaje de los estudiantes, ya que todos 
los docentes afirmaron no recibir acompañamiento pedagógico, es decir, ni las típicas supervisiones de la Secretaría de Educación, del director del centro educativo y de los coordinadores de las carreras y maestros del Bachillerato Técnico Profesional en Contaduría y Finanzas. Asimismo, otro docente afirmó que nunca un docente del área contable ha llegado a decirle profesor ensénele este tema a los alumnos para que lo desarrollen en un programa utilizando como recurso la computadora.

Sin embargo, un docente afirmó que a diferentes grupos de estudiantes les asigna un proyecto contable en el software Mónica 8.5. Al momento de la presentación y evaluación se invitan maestros de Contaduría y Finanzas con el propósito de valorar los aprendizajes de los estudiantes y de recibir procesos de retroalimentación que le ayude a mejorar su desempeño y el aprendizaje de los estudiantes. Hay que agregar que los docentes manifestaron tener necesidades de capacitación en conocimientos contables, uso y manejo de un software contable y una orientación del enfoque curricular que debe tener el módulo de Informática Contable.

\section{Elementos del Proceso Didáctico}

Figura 5

Elementos didácticos del proceso enseñanza aprendizaje en el módulo de Informática Contable

$90 \%$

$6 \%$

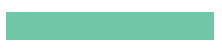

No aceptable
$4 \%$

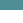

Indeciso

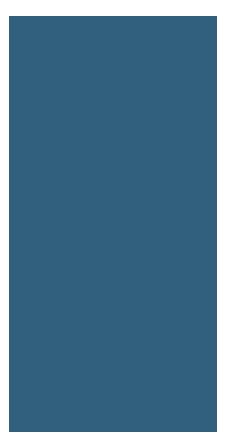

Aceptable 
Noventa por ciento (90\%) de los estudiantes manifestaron estar de acuerdo con la metodología de enseñanza y aprendizaje utilizada en el desarrollo del módulo de Informática Contable. Es decir, que las actividades de enseñanza y aprendizajes son ejercicios prácticos y demostrativos usando como recurso un software, asimismo, los estudiantes desarrollan las tareas o evaluaciones en las computadoras del laboratorio de computación y éstas consisten en prácticas contables; por último, manifiestan que el libro de texto, los materiales adicionales y la evaluación aplicada por el docente facilita el aprendizaje.

Sin embargo, al triangular datos con otros instrumentos se observó que el docente hace una planificación tradicional con un enfoque por competencias debido a que el planeamiento didáctico, únicamente se hace una jornalización de contenidos por semestre y un plan de unidad por cada parcial siguiendo los lineamientos curriculares de los programas de estudio del Bachillerato en Contaduría y Finanzas. Pero si consideramos los datos de la Tabla 2, se puede decir que muchos lineamientos curriculares no se logran, debido a que algunos institutos no cuentan con recursos transcendentales, entre ellos un software contable y el programa donde se hace la declaración de tributos e impuestos, lo que impide que los estudiantes logren aprendizajes muy importantes para la formación de las competencias del módulo curricular.

La técnica de enseñanza más utilizada por el docente en la exposición magistral, la cual inicia con una etapa organizativa donde se observa y se verifica que los estudiantes tengan todos los recursos necesarios para iniciar la clase. El docente utiliza un proyector multimedia, videos tutoriales, materiales adicionales y su computadora personal, después explica cada instrucción y el docente les va demostrando y proyectando en su computadora personal como se debe ir haciendo los procedimientos en la computadora donde está trabajando el estudiante, si el alumno realiza consultas sobre algunas dudas el docente repite otra vez el proceso usando los recursos como la computadora personal y el proyector multimedia. En la etapa organizativa y de desarrollo, el docente observa y se percata que el estudiante esté desarrollando los procedimientos y reciben asistencia personalizada cuando el estudiante 
la requiere. Durante este proceso las actividades de enseñanza que al mismo tiempo son las actividades de aprendizajes terminan siendo una actividad de evaluación en la cual algunos docentes verifican que el estudiante haya terminado y lo registra en el control porque le asignará una calificación.

Las tareas o actividades del estudiante están centradas en la enseñanza y otras en el aprendizaje, con principal énfasis en herramientas, reportes y registros de tipo contable financiero, que se tienen que desarrollar en los programas informáticos que dispone cada institución. Los docentes utilizan los recursos o medios que están disponible en su entorno como ser computadoras personales, proyector multimedia, computadoras del laboratorio de computación, puntero, equipo de sonido, software, manuales, videos tutoriales, materiales adicionales y libros de informática; los docentes que tienen acceso a Internet utilizan videos de YouTube, nubes de almacenamiento y plataformas de mensajería. Es importante señalar que existe una carencia de recursos primordiales para lograr las competencias de los estudiantes entre ellos computadoras, mobiliario, software o programas, impresoras y libros de texto acorde a los lineamientos curriculares.

Los docentes aplican la evaluación diagnóstica para identificar algunas debilidades de los estudiantes y realizar procesos de retroalimentación, además, manifiestan aplicar criterios para evaluar una actividad de aprendizaje entre ellos la responsabilidad en la entrega de las tareas, orden y presentación y que lo resultados sean correctos y funcionales. Los instrumentos de evaluación aplicados por el docente son las pruebas objetivas que tienen un valor por cada parcial de $45 \%$ y consisten en ejercicios prácticos que los estudiantes desarrollan en la computadora, igualmente, utilizan la lista de asistencia, lista de cotejo sobre trabajos realizados en clase y el cuadro de acumulativos. Solamente un docente utiliza rúbricas de valoración numérica; en cada instrumento se determinan criterios para valorar y medir el nivel de desarrollo de capacidades, grado de aprendizaje adquirido y asimilación de contenidos, que le permita objetivamente al docente consignar una nota final por parcial o semestre. 


\section{Conocimientos Previos}

Figura 6

Conocimientos previos del estudiante antes de iniciar el módulo de Informática Contable $76 \%$

$21 \%$

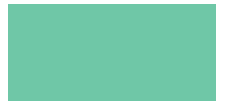

No aceptable
$3 \%$

Indeciso

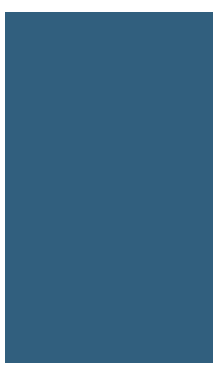

Aceptable

El estudio reflejó que un $76 \%$ de los estudiantes están de acuerdo que al iniciar el módulo de Informática Contable tienen habilidades y conocimientos en el uso de la computadora, dominio de Microsoft Excel, organizar archivos, buscar información en Internet, imprimir documentos y ciertos conocimientos en contabilidad. Estos conocimientos previos son la clave para la asimilación de los contenidos del módulo de Informática Contable debido a la relación que se establece entre los nuevos contenidos y las ideas ya existentes en la estructura cognitiva del estudiante, tal como se describe en la teoría de asimilación de Ausubel, Hanesian y Novak (1983, citado en Molina, 2005, p.86) donde "el factor más importante que influye en el aprendizaje es lo que el alumno ya sabe".

Según los docentes, antes de iniciar el módulo el estudiante debe tener "conocimiento básico en matemáticas relacionados a porcentajes, conversión de tasas y tiempo, interés simple y compuesto; conocimiento básico en el uso de la computadora, dominio en el manejo del paquete Microsoft Office (Excel y Word) y organizar archivos. Además, de conocimientos contables donde el estudiante pueda hacer un registro contable, conocer los movimientos de las cuentas y elaborar los Estados Financieros. 
Sin embargo, todos los docentes manifestaron que muchos estudiantes no traen conocimientos básicos en Informática y Matemáticas, situación que los obliga a realizar un proceso de retroalimentación utilizando un tiempo que posteriormente impide cumplir con el desarrollo del programa curricular.

\section{Número de Estudiantes por Sección}

La investigación refleja que hay institutos que tienen secciones con un número superior de estudiantes que lo establecido en la Ley Fundamental de Educación que son de 30 estudiantes por sección. Según la Tabla 1, se puede observar que hay institutos que disponen de menos computadoras en comparación al número de estudiantes, a pesar que tienen un número inferior de estudiantes a lo establecido en la Ley Fundamental de Educación, es decir, que no hay correspondencia entre número de estudiante por sección y la disponibilidad de mobiliario y equipo de informática para que cada estudiante pueda disponer de una computadora de forma individual y que pueda realizar las diferentes actividades de enseñanza y aprendizaje.

Esta situación conlleva a que los estudiantes deban compartir recursos con sus compañeros lo que puede generar posturas incorrectas al compartir mobiliario y desmotivación al compartir las computadoras. Además, se dificulta la atención individualizada, se atrasa los procesos de enseñanza, aprendizaje y evaluación; no se dispone de tiempo para procesos de retroalimentación, disminuye la interacción alumno - profesor, se desfavorece la visibilidad y cuesta mucho mantener el control de las diferentes conductas y comportamientos de los estudiantes.

\section{Los Horarios}

Un cien por ciento (100\%) de los estudiantes afirmaron recibir 6 horas clases a la semana que es lo que está establecido en el programa curricular, asimismo, todas las horas clases son recibidas en el laboratorio de computación. Sin embargo, en dos instituciones educativas los horarios no están estructurados ni organizados en bloques de dos horas clases consecutivas, esto genera una condición no favorable ya que el docente requiere 
de mucho tiempo para las actividades de enseñanza-aprendizaje y de evaluación, además, esto puede generar tiempo ocioso al cambio de las clases y que los estudiantes durante el día puedan estudiar varios módulos curriculares que no tengan relación alguna desde el punto de vista de los contenidos.

\section{Conclusiones}

Las conclusiones de esta investigación corresponden a un resumen del análisis de las nueve variables que responden a los objetivos de investigación sobre las condiciones de infraestructura, el perfil de los docentes que atienden el módulo de Informática Contable, las condiciones didácticas en que se desarrolla dicho módulo y la valoración que hacen los estudiantes sobre el desarrollo del módulo curricular desde la perspectiva de sus aprendizajes. Las conclusiones del presente estudio se enuncian a continuación.

Las condiciones de infraestructura no son las adecuadas, ya que la mayoría de los institutos tienen carencia en elementos de infraestructura o recursos didácticos (faltan computadoras, mobiliario, software contable, programa Det-Live, impresoras, y dispositivos de almacenamiento externo; existencia de problemas técnicos de almacenamiento temporal y el hecho que no hay acceso a Internet en los Laboratorios de Computación) lo que dificulta llevar a cabo el proceso didáctico y de evaluación de los aprendizajes; ante esta situación no se pueden desarrollar todas las competencias profesionales establecidas en el programa de estudio del módulo de Informática Contable.

En cuanto al perfil del docente, algunos de ellos no tienen la formación inicial docente en el área administrativa, contable y financiera; según el Manual de Clasificación de Puestos y Salarios Docentes de la Secretaría de Educación, lo que afecta el dominio teórico y metodológico, en cuanto a planificar, diseñar, facilitar, orientar, dirigir y evaluar actividades de aprendizaje en donde se utilice la tecnología para el registro de las transacciones generadas por las operaciones de tipo contable y financiera. Además, ningún docente participa en programas de formación o profesionalización, y el acompañamiento pedagógico no está cumpliendo con sus propósitos de mejora continua 
en los procesos de enseñanza aprendizaje, sin embargo, los docentes demuestran tener habilidades personales y manifiestan tener necesidades de capacitación en diferentes temáticas.

Las condiciones didácticas en que se desarrolla el módulo de Informática Contable no son favorables, debido a que los institutos no cuentan con suficientes recursos o medios didácticos para desarrollar las actividades de enseñanza aprendizaje y de evaluación de los contenidos que constituyen las competencias profesionales establecidas en el programa curricular. En la parte metodológica, la técnica de enseñanza más utilizada por los docentes es la exposición magistral de tipo demostrativo, en este sentido, los docentes desarrollan los contenidos haciendo uso del proyector multimedia, donde ellos explican las instrucciones y procedimientos a seguir para el desarrollo de las actividades que requieren el uso de la computadora. Los estudiantes realizan sus actividades de aprendizaje y tareas en las computadoras y programas disponibles en cada laboratorio, afectando aquellos estudiantes que trabajan en pareja debido a que algunos institutos no cuentan con suficientes computadoras, esto genera atrasos en los procesos de aprendizaje ya que los estudiantes debe compartir el equipo informático, sin embargo es pertinente decir que casi la mitad de los estudiantes (44\%) disponen de computadora en su casa y este recurso no está siendo aprovechado por los docentes, ya que los estudiantes manifiestan que muy pocas veces la utilizan por la falta de asignación de tareas para realizar en casa, razón que no se justifica ya que para lograr las competencias del programa curricular es obligatorio desarrollar las prácticas contables haciendo uso de la computadora y programas.

Se realiza evaluación diagnóstica, donde se identifican debilidades en cuanto a conocimientos básicos en informática y matemáticas, por lo que muchos estudiantes no tienen los conocimientos previos para asimilar los nuevos contenidos lo que obliga a realizar readecuaciones y retroalimentación de algunos contenidos. Asimismo, los docentes aplican pruebas objetivas como instrumento para valorar y medir el aprendizaje de contenidos conceptuales y procedimentales; y solamente un docente utiliza la rúbrica 
de valoración numérica como instrumento de evaluación. Finalmente, las secciones de estudiantes tienen un número superior a lo establecido legalmente y en relación a la cantidad de recursos informáticos disponibles.

La valoración que hacen los estudiantes sobre el desarrollo del módulo de Informática Contable desde la perspectiva de su aprendizaje es positiva con relación aspectos de infraestructura, perfil docente, elementos del proceso didáctico y de evaluación de los aprendizajes; no obstante, hay que tomar en cuenta que los estudiantes tienen un conocimiento limitado para emitir juicios objetivos sobre algunos elementos curriculares, metodológicos y didácticos que solamente el docente, como especialista posee, debido a su formación, capacitación y experiencia, lo cual se manifiesta en los resultados obtenidos en esta investigación.

\section{Propuesta}

Después de realizar un análisis en la investigación, se pudo identificar algunos problemas o dificultades de infraestructura en los centros educativos que de alguna manera afectan el desarrollo de los procesos de enseñanza aprendizaje del módulo de Informática Contable; además, se identificaron condiciones didácticas no favorables como ser la carencia de recursos o medios didácticos que impiden el desarrollo de una serie de actividades para concretar el aprendizaje de contenidos que constituyen las competencias profesionales; y por último, en el estudio se identificaron algunas debilidades en el desempeño del docente que atiende el módulo.

Pues ante esta situación, resulta pertinente proponer una serie de estrategias con el fin de superar las dificultades o problemas de infraestructura, condiciones didácticas no favorables y debilidades en el desempeño docente; teniendo como único propósito mejorar la calidad de los procesos de enseñanza aprendizaje en el módulo de Informática Contable. A continuación, se presentan las estrategias sobre condiciones de infraestructura, estrategias sobre condiciones didácticas y de evaluación, y finalmente, las estrategias para mejorar las habilidades y competencias del docente que atiende el módulo curricular. 


\section{Estrategias Propuestas sobre Condiciones de Infraestructura}

1. Gestionar con toda la comunidad educativa la adquisición de computadoras y mobiliario, para que cada estudiante disponga de una computadora de forma individual. Adquirir impresoras que estén disponibles en los laboratorios de computación con el fin de imprimir documentos que sirvan para hacer procesos de aprendizajes en el uso de la tecnología, retroalimentación y autoevaluación de las actividades desarrolladas. Instalar en los laboratorios de computación equipo de sonido para reproducir videos tutoriales e instalar los proyectores en lugares estratégicos que permita una excelente visibilidad.

2. Gestionar que los Laboratorios de Computación tengan acceso a Internet, esto permite utilizar antivirus de manera gratuita para darle mantenimiento a las computadoras y eliminar virus que afectan el funcionamiento de las mismas sin necesidad de estar programadas con almacenamiento temporal. También permite acceder a medios didácticos como videos tutoriales y descargar los programas como Mónica 8.5, Express Acounts, DET - Live; acceder a aplicaciones de mensajería y a utilizar nubes de almacenamiento. Por último, los laboratorios deben tener instalados todos los programas que se necesitan en el módulo curricular y esta debe ser responsabilidad del docente y del asistente del laboratorio en aquellos institutos que disponen de este recurso humano.

3. Se recomienda a las autoridades de la Secretaría de Educación y al personal directivo de los institutos no tener una matrícula superior a los 30 estudiantes por sección para garantizar mayores oportunidades de aprendizaje al estudiante según (Dockendorff, 2015). Además, considerando que los institutos tienen como mínimo dos Laboratorios de Computación y personal docente de la especialidad de Educación Comercial, se pueden hacer procesos de reubicación docente donde se pueden separar las secciones en dos grupos de estudiantes y crear un horario paralelo en bloque de dos horas clases (Marín \& Maya, 2015) para que cada grupo reciba las clases de Informática Contable y se le asigne estratégicamente un laboratorio de computación y un docente del área de Educación Comercial. 


\section{Estrategias Didácticas y de Evaluación en el Módulo de Informática Contable.}

En el programa de estudio del módulo de Informática Contable se establece ciertos criterios de desempeño y se sugieren algunas actividades de enseñanza y aprendizaje para lograr las competencias profesionales del módulo curricular (PRAEMHO, 2007, p. 548). La investigación refleja que la mayoría de los institutos no utilizan el Software Mónica 8.5, indicado en el programa de estudio, además, ninguno de ellos utiliza otro software contable ni la página electrónica de declaración de tributos; por lo tanto, podemos decir que en los procesos de enseñanza aprendizaje del módulo de Informática Contable no se desarrollan las estrategias o actividades establecidas en el programa de estudio para lograr los criterios de desempeño o competencias profesionales. Para cumplir y seguir los lineamientos establecidos en el currículo resulta importante proponer las siguientes estrategias:

1. Hacer un proceso de retroalimentación para enseñarle al estudiante administrar archivos, donde aprenda a copiar, pegar y guardar archivos en la computadora y dispositivos de almacenamiento.

2. Los docentes deben crear actividades de enseñanza y aprendizaje para que los estudiantes usen su computadora personal y las computadoras del Laboratorio de Cómputo. Se debe organizar y concientizar a los alumnos para que puedan compartir su computadora personal con sus compañeros de curso.

3. Enseñar a los estudiantes a instalar y desinstalar el software contable Mónica 8.5, Express Acounts, respaldar carpetas de información del programa; restaurar, copiar y pegar carpetas de información del programa. Estos aprendizajes le permitirán al estudiante resolver algunos problemas técnicos sin la necesidad de ayuda por parte del docente, lo que le permitirá trabajar en cualquier computadora que el alumno decida.

4. La primera actividad que se recomienda realizar en el programa Mónica 8.5 es crear o hacer 35 asientos contables, posteriormente se debe extraer en archivos PDF los respectivos reportes contables. Para esta actividad el docente debe de entregar todos los recursos que se necesitan y llevar una secuencia didáctica de cada procedimiento, es recomendable que esta actividad se desarrolle por completo en el aula de clase, en caso de que los alumnos cometan errores, se debe hacer una corrección y retroalimentación 
de los mismos. Se debe de utilizar instrumentos de evaluación como lista de cotejo o rúbricas de valoración numérica donde se evaluarán los criterios de desempeño establecidos.

5. La segunda actividad que se recomienda realizar en el programa Mónica 8.5 es crear un catálogo de 140 cuentas de activo, pasivo, patrimonio, ingresos, costos y gastos. Después crear como mínimo 20 asientos contables y extraer en archivo PDF los reportes contables. El docente debe facilitar un archivo que contenga las instrucciones sobre la creación del catálogo y los respectivos asientos contables. Debe dar las indicaciones y llevar una secuencia didáctica de cada procedimiento y una atención individualizada cuando el estudiante lo requiera. Se debe utilizar instrumentos como la lista de control o rúbrica de valoración numérica donde se lleve un control de los criterios de desempeño establecidos para esta actividad.

6. La última actividad a realizar en el programa Mónica es un laboratorio contable que se debe presentar en un informe de manera digital o impreso. El docente debe facilitar un archivo que contenga todas las instrucciones y una secuencia didáctica desde el asiento inicial, enlace contable, creación del inventario, creación de la cartera de clientes y proveedores, creación de cuentas bancarias, ordenes de compras a realizar, pagos a proveedores, facturas de ventas y cobros a realizar. Una vez finalizado el laboratorio el docente debe de dar instrucciones de los archivos que se van a extraer y la forma como se van a presentar el informe. Se sugiere utilizar una rúbrica de valoración numérica donde se establezcan criterios de desempeño en cuanto a resultados, ya que los valores de los reportes contables tienen que coincidir con los informes de cada módulo de actividad o funciones del programa. Se tiene que hacer un proceso de retroalimentación y autoevaluación para que los estudiantes puedan compartir su experiencia de aprendizaje y aporten recomendaciones que faciliten regulaciones proactivas en próximas actividades.

7. Se sugiere utilizar el software contable Express Acounts ya que puede procesar y presentar mayor cantidad de información contable y financiera, con el agregado, de que la contabilidad esta enlazada y es de fácil manipulación. En este programa contable se sugiere realizar las estrategias 4, 5 y 6 de este apartado, donde el docente debe de realizar las adaptaciones y readecuaciones a las características propias del programa. 
8. En la página electrónica de declaración de tributos DET Live se recomienda realizar cinco declaraciones juradas de impuesto sobre la renta y declaración de impuesto sobre ventas. El docente debe facilitar un instructivo con la información general y contable para realizar la declaración, donde los datos y la información contable tiene que coincidir y no tener errores, ya que los resultados de la declaración deben coincidir con algunos de los datos contables presentados. Se recomienda que el docente explique demostrativamente una declaración de impuesto sobre la renta e impuesto sobre ventas, y que al finalizar cada declaración el docente señale que los resultados coinciden con los valores contables que tienen a la fecha los impuestos a pagar. Después el estudiante debe de realizar el resto de las declaraciones siguiendo los procedimientos enseñados por el docente. En la evaluación se recomienda utilizar rúbricas de valoración numéricas, donde se pueden evaluar si los resultados de la declaración de impuesto coinciden con la información contable facilitada. Es necesario realizar proceso de retroalimentación y autoevaluación para compartir experiencias y obtener recomendaciones para próximas actividades de aprendizaje.

9. Se debe realizar una evaluación diagnóstica para identificar dificultades o habilidades del estudiante con relación algunas habilidades; y tener sustento para realizar la readecuación o retroalimentación que lleve a que los alumnos obtengan los conocimientos necesarios que les permita desarrollar con facilidad algunas actividades en Microsoft Excel.

10. Una de las herramientas financieras para la toma de decisiones a corto, mediano y largo plazo que se pueden hacer en Excel es el presupuesto maestro. El docente debe organizar a los estudiantes y darle instrucciones claras y precisas de la información que necesitan para realizar presupuesto maestro, además, cuando el alumno individualmente presente la información solicitada, el docente debe revisarla para valorarla y hacer las respectivas correcciones. Después el docente haciendo uso de la computadora y del proyector explicará paso a paso un presupuesto maestro y el estudiante individualmente irá realizando el presupuesto con la información que le solicito el docente. Una vez terminado el presupuesto se puede hacer demostraciones sobre los efectos financieros y la toma de decisiones que se tiene que hacer en caso que disminuyen a aumenten algunos costos, pues bastaría solo cambiar unos datos y el presupuesto permite ver 
automáticamente los efectos financieros, por lo que esta herramienta facilita la toma de decisiones. El docente debe guiar, orientar y dar atención individualizada durante todo el proceso, se puede utilizar una lista de cotejo para valorar todo el proceso y el aprendizaje del estudiante.

11. La Secretaría de Educación debe gestionar la elaboración de un libro de texto que incluya todos los contenidos curriculares y organizados jerárquicamente según el plan de estudios. Se recomienda que todas las estrategias didácticas y de evaluación descritas en este apartado deben estar incluidas en la estructura del libro de texto y que su aprobación se haga en base a los elementos actuales de nuestro currículo.

\section{Estrategias Propuestas para Mejorar las Habilidades y Competencias del Docente.}

1. Realizar un proceso de formación y capacitación técnica para los docentes del área de Educación Comercial, coordinadores de área y personal técnico pedagógico de la Secretaría de Educación del Municipio de El Progreso, Yoro. El programa debe consistir en el uso y manejo de programas como Microsoft Excel, Software Contable Mónica 8.5, página electrónica de declaración de tributos DET-Live y el Software Contable Express Acounts; durante el proceso se desarrollarán estrategias de enseñanza, aprendizaje y de evaluación; con el fin de que el docente desarrolle las competencias que le permita planificar, diseñar y aplicar estrategias didácticas o de evaluación utilizando cada uno de los programas informáticos. Se recomienda que participen todos los docentes de Educación Comercial independientemente que no imparta el módulo de Informática Contable, porque los aprendizajes adquiridos le permitirán diseñar estrategias para implementar las TIC en los módulos curriculares como Contabilidad y Administración Financiera.

2. En el proceso de formación técnica se pueden planificar y ejecutar las estrategias didácticas y de evaluación propuestas en este apartado, ya que estas estrategias se han propuesto con el objetivo de facilitar los procesos de enseñanza y aprendizaje en el módulo de Informática Contable.

3. Planificar y ejecutar un acompañamiento pedagógico que este coordinado y dirigido con recurso humano que haya participado en el proceso de capacitación y de esta 
manera apoyen a los docentes dándoles asesoría, intercambiar ideas, proponer nuevas técnicas y recursos didácticos, analizar situaciones, hacer críticas constructivas y readecuar algunos elementos del currículo.

4. Se le sugiere a las autoridades de la Secretaría de Educación y al personal directivo de los institutos, que el módulo de Informática Contable sea asignado a docentes que tienen una formación inicial en Educación Comercial y no a los de Informática Educativa u otra área, siguiendo lo establecido en el Manual de Puestos y Salarios Docentes de la Secretaría de Educación, debido a que el docente en Educación Comercial tiene una formación en el área administrativa, contable, financiera y manejo de las TIC que le facilita planificar, organizar, dirigir, ejecutar y evaluar procesos de enseñanza aprendizaje en el módulo de Informática Contable. Además, el proceso de capacitación propuesto es viable para el Profesor de Educación Comercial; y no para el de Informática Educativa que necesita centrarse en conocimientos administrativos, contables y financieros, áreas que son muy extensas y que necesita de mucho tiempo, por lo tanto, tendrían que participar en procesos de formación formal en estas áreas del conocimiento.

\section{Referencias}

Carrasco, L. (2009). Formación y acompañamiento docente. Santo Domingo: Editorial Corripio. Recuperado el 10 de Febrero de 2018, de http://www.feyalegria.org/images/ acrobat/Formacion_y_AcompanamientoDocente.pdf

Castro, M., \& Morales, M. E. (2015). Los ambientes de aula que promueven el aprendizaje, desde la perspectiva de los niños y niñas. Revista Electrónica Educare, 1-32. doi: http://dx.doi.org/10.15359/ree.19-3.11

Coll, C. S. (1992). Concepción constructivista y planeamiento curricular. España: Santillana.

Díaz, F. A., \& Hernández, F. (2007). Estrategia docente para un aprendizaje significativo. Recuperado el 30 de Mayo de 2019, de http://formacion.sigeyucatan.gob.mx/formacion/ materiales/4/4/d1/p1/2.\%20estrategias-docentes-para-un-aprendizaje-significativo.pdf 
Díaz, Á. (2005). Evaluación curricular y evaluación de programas con fines de acreditación. Cercanías y desencuentros. Recuperado el 06 de Mayo de 2019, de http://www. angeldiazbarriga.com/ponencias/conferencia_cnie2005.pdf

Díaz, F. A., \& Hernández, F. (2007). Estrategia docente para un aprendizaje significativo. México: McGraw-Hill. Recuperado el 30 de Mayo de 2019, de http://formacion. sigeyucatan.gob.mx/formacion/materiales/4/4/d1/p1/2.\%20estrategias-docentespara-un-aprendizaje-significativo.pdf

Dockendorff, M. E. (2015). Número de alumnos por curso: un estado del arte. Tesis de Postgrado, Pontífica Universidad Católica de Chile, Santiago, Chile. Recuperado el 25 de Febrero de 2019, de https://repositorio.uc.cl/bitstream/handle/11534/15783/000656411. pdf? sequence $=1$

Fernández, A. (2010). La evaluación de los aprendizajes en la universidad: nuevo enfoque. Recuperado el 1 de Junio de 2019, de https://webcache.googleusercontent. com/search?q=cache:a-1s04WSvaQJ:https://web.ua.es/es/ice/documentos/recursos/ materiales/ev-aprendizajes.pdf $+\& \mathrm{~cd}=1 \& \mathrm{hl}=\mathrm{es}-419 \& \mathrm{ct}=\mathrm{clnk} \& \mathrm{gl}=\mathrm{hn}$

Flores, R., Castro, J. A., Galvis, D. J., Acuña, L. F., \& Zea, L. A. (2017). Ambientes de aprendizaje y sus mediaciones. Bogotá: IDEP- Investigación Educativa y el Desarrollo Pedagógico.

Hernández, S. R., Fernández, C. C., \& Baptista, L. P. (2014). Metodología de la Investigación. México: McGraw-Hill Educatión.

Marín, J. C., \& Maya, P. A. (2015). Modelo lineal para la programación de clases en una institución educativa. Ingeniería y Ciencia, 47- 71. doi:0.17230/ingciencia.12.23.3

Mattos, L. (1974). Compendio de didáctica general. Buenos Aires: Kapelusz.

Medina, A., \& Mata, F. S. (2009). Didáctica General. Madrid: PEARSON EDUCACIÓN.

Meza, J. L. (2012). Diseño y desarrollo curricular. México: Red Tercer Milenio S. A. Recuperado el 15 de Junio de 2018, de https://es.scribd.com/doc/261419367/Disenoy-Desarrollo-Curricular 
Molina, S. (2005). Los conocimietos previos de los estudiantes del tercer curso de Magisterio acerca de la Organización Escolar: implicaciones para la docencia universitaria. Aula Abierta, 85, 85-104. Recuperado el 19 de Septiembre de 2020, de file://C:/Users/jeffr/ Downloads/Dialnet-LosConocimientosPreviosDeLosEstudiantesDeTercerCur-2044872. pdf

Nakazawa, F., García, A. M., Calderas, T., Alanis, J. R., Olivares, C., Uribe, A., \& Campos, C. (2018). Condiciones básicas para la enseñanza y aprendizaje en los planteles de educacón media superior en México. México: INEE. Recuperado el 10 de Mayo de 2019, de https://www.google.com/

Perdomo, C. R. (2016). Casimirus o de los diálogos sobre la reforma educativa. San Pedro Sula, Honduras: INVERSAFE.

Perdomo, N. A. (2013). El acompañamiento docente de parte de la Unidad de supervisión de la Dirección Departamental de Educación de Ocotepeque. Tesis de Postgrado, UPNFM. Recuperado el 24 de Mayo de 2019, de https://www.google.com/

Pérez, C. (2004). Técnica de Análisis Multivariante de Datos. Madrid: PEARSONEDUCACIÓN. Recuperado el 27 de Agosto de 2017, de http://www.ub.edu/stat/ personal/cuadras/metodos.pdf

Pico, E. V., \& Núñez, S. C. (2018). El software contable como herramienta técnica en las microempresas de la provincia Santa Elena, Ecuador. Revista Killkana Sociales, 21-26. doi:https://doi.org/10.26871/killkana_social.v2i1.242

Pimienta, J. (2007). Metodología constructivista. México: PEARSON EDUCACIÓN.

Pimienta, J. (2008). Evaluación de los aprendizajes: enfoque por competencias. México: PEARSON EDUCACIÓN. Recuperado el 27 de Mayo de 2019, de https://josedominguezblog.files.wordpress.com/2014/08/evaluacic3b3n-de-losaprendizajes-enfoque-basado-en-competencias.pdf

Pimienta, J. (2012). Estrategias de enseñanza-aprendizaje. México: PEARSON EDUCACIÓN. Recuperado el 2 de Junio de 2019, de http://web.uaemex.mx/ incorporadas/docs/MATERIAL\%20DE\%20PLANEACION\%20INCORPORADAS/ SD\%20Estrategias\%20de\%20ensenanza-aprendizaje.pdf 
PRAEMHO. (Noviembre de 2007). Planes y programas de estudio de educación media. Recuperado el 21 de Agosto de 2017, de https:/www.se.gob.hn/media/files/educmedia/ documentos/doc/Auerdo_15154_y_15155-SE-2012-BTP.pdf

Secretaría de Educación. (2017). Manual de Clasificación de Puestos y Salarios. Tegucigalpa. Recuperado el 18 de Noviembre de 2018, de https:/sace.se.gob.hn/media/comunicados/ manual_puestos_docentes_oct_2017.pdf

Semenov, A. (2005). Las Tecnologías de la Informción y Comunicación en la Enseñanza. Paris: División de Educación Superior (UNESCO). Recuperado el 15 de Noviembre de 2018, de http://unesdoc.unesco.org/images/0013/001390/139028s.pdf

Tobón, S. (2005). Formación Basada en Competencias. Bogotá: ECOE- EDICIONES.

Torres, M. H., \& Girón, D. A. (2009). Didáctica General. San José, Costa Rica: Coordinación Educativa y Cultural Centroamericana.

Valzacchi, J. (2003). Internet y educación: aprendiendo y enseñando en los espacio virtuales. Buenos Aires: INTERAMER. Recuperado el 13 de Mayo de 2019, de http://www. educoas.org/portal/bdigital/contenido/valzacchi/ValzacchiCapitulo-19New.pdf 
50 <Paradigma> Revista de Investigación Educativa | Año 27 | N44 | Julio-Diciembre 2020 | ISSN: 1817-4221 | EISSN: 2664-5033

(c) (7) $\odot$ Los artículos de la Revista Electrónica Paradigma del Instituto de Investigación y Evaluación Educativas y Sociales de la Universidad Pedagógica Nacional cc) Fr NC ND Francisco Morazán, Honduras, se comparten bajo términos de la Licencia Creative Commons: Reconocimiento, No Comercial, Sin Obra Derivada 